Research Article

Cellular, Molecular and Developmental Genetics

\title{
Periostin regulates osteogenesis of mesenchymal stem cells from ovariectomized rats through actions on the ILK/Akt/GSK-3 $\beta$ Axis
}

\author{
Silin Liu ${ }^{1,2}$, Zuolin Jin ${ }^{1}$, Meng Cao ${ }^{1}$ (D), Dandan Hao ${ }^{3}$, Chunrong $\mathrm{Li}^{1}$, Doudou $\mathrm{Li}^{1}$ and Weiwei Zhou ${ }^{1}$ \\ ${ }^{\prime}$ The Fourth Military Medical University, School of Stomatology, Department of Orthodontics, \\ State Key Laboratory of Military Stomatology \& National Clinical Research Center for Oral Diseases \\ \& Shaanxi Clinical Research Center for Oral Diseases, Xi'an, China. \\ ${ }^{2}$ Affiliated Hospital of Chifeng University, Department of Orthodontics, Inner Mongolia, China. \\ ${ }^{3}$ Chifeng University, Medical College, Department of Physiology, Inner Mongolia, China.
}

\begin{abstract}
Osteoporosis is a condition of the skeleton that mainly results from estrogen deficiency. Periostin is a matricellular component in bone that is involved in osteoblast differentiation. However, how Periostin promotes osteogenesis remains largely unknown. Here, we isolated bone marrow skeletal stem cells (BMSCs) derived from an ovariectomy (OVX)-induced osteoporosis rat model and the effects of periostin on BMSCs derived from OVX rats (OVX-BMSCs) were assessed. Overexpression of periostin enhanced alkaline phosphatase (ALP) and alizarin red staining in OVXBMSCs as well as the osteogenic genes OCN, BSP and Runx2. ILK is a downstream effector of signals from the extracellular matrix and participates in bone homeostasis. Overexpression of periostin also increased expression of protein levels for ILK, as well as the downstream targets pAkt and pGSK3 $\beta$. Suppression of ILK or Akt partially suppressed the enhancement of osteogenic ability induced by periostin overexpression in OVX-BMSCs. Thus, periostin may promote the osteogenic ability of OVX-BMSCs through actions on the ILK/Akt/GSK3 $\beta$ axis.
\end{abstract}

Keywords: Osteoporosis, periostin, osteoblast, bone marrow skeletal stem cells, osteogenesis.

Received: December 10, 2020; Accepted: June 01, 2021.

\section{Introduction}

About $50 \%$ of postmenopausal women worldwide suffer from osteoporosis, a common condition of the skeleton (Eastell et al., 2016), with estrogen deficiency being one of its major causes (Friedlander, 2002). Bone is actively remodeled throughout life, which is accomplished by interactions between osteoclasts and osteoblasts. Osteoclasts drive bone resorption and osteoblasts produce new bone matrix. The osteoblast is differentiated from bone marrow stromal cells/skeletal stem cells (BMSCs), which can also differentiate into adipocytes. The switch to adipogenesis or osteogenesis is critical for $\mathrm{BMSC}$ in bone regeneration i.e. BMSCs can differentiate into adipocytes at the expense of osteoblasts and vice versa (Gimble et al., 2006).

Osteoporosis biopsies from humans have revealed the replacement of bone mass by adipose tissue (Meunier et al., 1971). It is well established that the commitment of BMSC to an osteoblast lineage is under tight regulation. A high dose of TGF $\beta /$ BMPs is essential for the expression of runt-related gene 2 (Runx2), which is an osteoblast-specific marker (Chen et al., 2012). Furthermore, Hedgehog signaling can interact with BMP signaling to promote osteoblast differentiation (Spinella-Jaegle et al., 2001). Wnt3a (Byun et al., 2014) and Wnt10b (Bennett et al., 2007; Stevens et al., 2010) also

Send correspondence to Meng Cao. The Fourth Military Medical University, School of Stomatology, Department of Orthodontics, State Key Laboratory of Military Stomatology \& National Clinical Research Center for Oral Diseases \& Shaanxi Clinical Research Center for Oral Diseases, 169 Changle West Road, Xi'an, China. E-mail: $1307753550 @ q q . c o m$. promote osteoblast-specific differentiation. On the other hand, Notch signaling has been reported to promote both adipogenic and osteoblast differentiation through disparate mechanisms (Ross et al., 2004; Deng et al., 2008; Song et al., 2015). Several cell line-based studies have revealed that estrogen deficiency impairs osteogenic differentiation. In mice, estrogen receptor- $\alpha(E R \alpha)$ is upregulated by Wnt3a overexpression and thus ER signaling together with Wnt3a can promote osteogenic differentiation (Gao et al., 2013).

Periostin, a member of the matricellular family, serves a scaffold function for extracellular matrix (ECM) protein assembly and can also bind to cell surface receptors such as integrin and Notch-1. Periostin is not only an organizer of ECM, but also a regulator of cell adhesion and differentiation. Thus, it plays essential roles in several diseases including fibrosis (O'dwyer and Moore, 2017), inflammatory disease (Koh et al., 2019; Ohno et al., 2019; Ryu and Kim, 2020) and tumorigenesis (Ma et al., 2019; Ma et al., 2020), and also regulates bone regeneration ( $\mathrm{Li}$ et al., 2019). Studies have revealed that ECM proteins and matricellular proteins including Periostin are upregulated after bone injury (Duchamp de Lageneste $e t$ al., 2018; Heo et al., 2015). Research on periostin KO mice has suggested a role for periostin in bone development and homeostasis (Norris et al., 2007; Bonnet et al., 2013). In a previous study, Periostin has been shown to participate in osteoblast differentiation through $\mathrm{Wnt} / \beta$-Catenin signaling (Bonnet et al., 2012; Zhang et al., 2017), which suggests that Periostin may be a novel target for osteoporosis therapy.

To investigate further the role of Periostin in osteogenic differentiation in an estrogen-depleted state, female rats were 
treated with ovariectomy (OVX) to induce postmenopausal osteoporosis and then BMSCs were isolated. We found that overexpression of periostin could promote osteogenesis of OVX-rBMSCs and this facilitation is likely accomplished through actions on the ILK/Akt/GSK3 $\beta$ axis.

\section{Material and Methods}

\section{Animals and establishment of the osteoporosis model}

All animals were purchased from the laboratory animal center of the PLA Air Force Military Medical University. Animal use and care protocols were conducted according to the Guide for the Welfare Committee of Laboratory Animals of the PLAAir Force Military Medical University (IRB approval number: 20170604). Six-week-old female Sprague-Dawley rats weighting $110 \pm 10 \mathrm{~g}$ were randomly assigned into OVX $(n=12)$ or sham groups $(n=12)$. Rats in the OVX group were subjected to bilateral ovary removal. Rats in the sham group were subjected to a sham operation (i.e., without removal of the ovaries). All the rats were bred for another 3 months before being humanely euthanized with carbon dioxide.

\section{MicroCT analysis}

To identify the establishment of the osteoporotic rat model, specimens were scanned with a microCT system (Siemens, Inveon MicroCT). All images were obtained using Inveon Research Workplace (Siemens).

\section{Cell isolation, culture and induction of differentiation}

Primary BMSCs were isolated from the femur of rats by flushing the bone marrow with DMEM (Gibco) supplemented with $1 \%$ FBS (Gibco) and 1\% penicillin-streptomycin solution (Gibco). Cells were then incubated at $37^{\circ} \mathrm{C}$ with added $5 \%$ gaseous $\mathrm{CO}_{2}$ and $95 \%$ humidity. The medium was refreshed every 3 days. After the cells reached $80-90 \%$ confluence, they were digested with $0.25 \%$ trypsin (Gibco) and passaged at a ratio of $1: 3$

For identification of BMSC markers, the third passage of BMSCs was subjected to flow cytometry analysis. Briefly, cells that reached $80 \%$ confluence were trypsinized and incubated with CD29 (eBioscience, 11-0291-80), CD34 (Santa Cruz, sc-19587), CD45 (Abcam, ab10558), CD90 (Bio-Rad, MCA47GA), followed by analysis using flow cytometry (CytoFLEX, Beckman Coulter). For each sample, 10,000 events were analyzed.

For osteogenic induction, the third passage of BMSCs were seeded in 6-well plates at a density of $2 \times 10^{5}$ cells/ well and cultured in DMEM containing 10\% FBS, $10 \mathrm{mM}$ $\beta$-glycerophosphate (Sigma), $0.1 \mu \mathrm{M}$ dexamethasone (Sigma) and $50 \mathrm{mg} / \mathrm{L}$ ascorbic acid (Sigma). The medium was refreshed every 3 days.

For adipogenic induction, the third passage of BMSCs were seeded in 6-well plates at a density of $2 \times 10^{5} /$ well and cultured in DMEM containing 10\% FBS, $10 \mu \mathrm{M}$ dexamethasone (Sigma), $200 \mu \mathrm{M}$ isobutylmethylxanthine (Sigma), 0.5 mM IBMX and $10 \mathrm{mg} / \mathrm{L}$ insulin (Sigma). The medium was refreshed every 3 days.

\section{Cell transfection and Akt/GSK-3 $\beta$ signaling modulation}

The recombinant lentiviral vector containing periostin was prepared by cloning full length rat periostin cDNA. The PCR primers for periostin cloning were as follows:

(5' - 3'): Forward: AGGTCGACTCTAGAGGATC CCGCCACCATGGTTCCTCTCCTGCCCTTATC, Reverse: TCCTTGTAGTCCATACCCTGAGAACGGCCTTCTCTT GATCGCCTTCTAGACCCTTGAACCCTTTTGTTG.

The resulting cDNA was digested with the BamHI/ AgeI restriction enzyme and then inserted into the vector GV492 (Genechem). The recombinant lentiviral vector and LentiEasy Packaging Mix (Genechem) were co-transfected into $293 \mathrm{~T}$ cells using Lipofectamine 2000 (Thermo Fisher) to generate the lentivirus.

Passage 3 of primary BMSCs were seed into 12-well plates at a density of $2 \times 10^{5}$ cells/well and incubated overnight. Then, the cells were transfected with $10 \mu \mathrm{L}\left(2.5 \times 10^{8} \mathrm{TU} / \mathrm{mL}\right)$ recombinant lentiviral vector or empty vector (multiplicity of infection, $\mathrm{MOI}=50$ ). After a $12 \mathrm{~h}$ incubation, the medium was replaced with regular medium and incubated for another $48 \mathrm{~h}$ followed by subsequent experiments. The transfection efficiency was detected by qPCR.

For inhibition of Akt/GSK-3 $\beta$ in BMSCs overexpressed with periostin, cells were transfected with recombinant lentiviral vector in the presence of $0.5 \mu \mathrm{M}$ Akt or GSK-3 $\beta$ inhibitor. The ILK inhibitor used was OSU-T315 (MCE, HY18676) and the Akt inhibitor genistein (MCE, HY-14596). After a $12 \mathrm{~h}$ incubation, the medium was replaced with regular medium and incubated for another $48 \mathrm{~h}$ and then subsequently experiments were conducted.

\section{Alkaline phosphatase (ALP), alizarin red and oil red O staining}

BMSCs derived from the Sham or OVX groups were seeded on 24-well plates at 1,000 cells/well for $24 \mathrm{~h}$. According to the grouping, cells were untreated or transfected with the corresponding recombinant lentiviral vectors (vide supra).

For ALP staining, cells after 7-days induction were fixed in 4\% formaldehyde (Sinopharm) for $15 \mathrm{~min}$ and then washed 3 times with distilled water. Then the cells were incubated with BCIP/NBT Chromogen Kit (Solarbio) and Nuclear fast red (Sinopharm) according to the manufacturer's protocol. Quantitative ALP activity was assessed using an alkaline phosphatase assay kit (Beyotime) according to the manufacturer's protocol. Briefly, cells were lysed with lysis buffer (1\% Triton X-100). Optical density was measured using a spectrophotometer (Thermo Fisher Scientific, Multiskan MK3) at $405 \mathrm{~nm}$ with p-nitrophenyl phosphate (p-NPP) (Sigma) as the substrate. Relative ALP activity was normalized to the total protein curve.

For alizarin red staining, cells after 21-days induction were fixed in $4 \%$ formaldehyde (Sinopharm) for $15 \mathrm{~min}$ and then incubated with alizarin red $\mathrm{S}$ solution $(0.2 \%, \mathrm{pH}$ 4.2, Solarbio) according to the manufacturer's protocol. For quantification of the relative degree of mineralization, the deposits were dissolved in 10\% hexadecylpyridinium chloride 
monohydrate (Sigma) solution for 5-10 min. The supernatant was collected and centrifuged at 2,000 rpm for $5 \mathrm{~min}$. Then the absorbance of $100 \mu \mathrm{L}$ supernatant was measured at 520 nm using a UV spectrophotometer. The results of ARS are expressed as $\mathrm{OD}_{520}$ with the mean \pm standard deviation.

For oil red O staining, cells after a 14-day induction were fixed in $4 \%$ formaldehyde (Sinopharm) and washed with $60 \%$ isopropanol. Next, cells were incubated with oil red O (Sigma) followed by staining with Mayer hematoxylin (Sigma) for 1-3 min.

Samples were observed with a phase-contrast microscope (Olympus, BX53) and images captured using cellSens (Olympus).

\section{RNA extraction and quantitative real-time PCR}

Total RNA was isolated with Trizol reagent (Ambion) and reverse-transcribed into cDNA with HiScript ${ }^{\circledR}$ Reverse Transcriptase (VAZYME) according to the manufacturer's instructions. For real-time PCR, a total of $20 \mu \mathrm{L}$ of reaction mixture containing $10 \mu \mathrm{L}$ of SYBR Green Master Mix (VAZYME), $0.4 \mu \mathrm{L}$ of $50 \times$ ROX Reference Dye 2 (VAZYME), cDNA and primers was incubated in an QuantStudio ${ }^{\text {TM }} 6$ Flex system (ABI). The primers (from 5' to 3') are listed in Table 1.

\section{Western blotting}

Total proteins were isolated using RIPA lysis buffer with added PMSF and phosphatase inhibitors (BEYOTIME), and were quantified using a BCA protein assay kit (BEYOTIME). The proteins were separated using $12 \%$ SDS-polyacrylamide gel electrophoresis and transferred to PVDF membranes (Millipore). After being blocked with 5\% fat-free milk, the membranes were incubated with primary antibodies overnight at $4{ }^{\circ} \mathrm{C}$. The primary antibodies used were rabbit anti-Periostin (Abcam, ab14041, 1:1000), mouse anti- $\beta$ actin (BOSTER, BM0627, 1:200), rabbit anti-ILK (Abcam, ab76468, 1:1,000), rabbit anti-pAkt (CST, 4060, 1:2,000), rabbit anti-Akt (CST, 4691, 1:1,000), rabbit anti-pGSK3 $\beta$ (Abcam, ab68476, 1:1,000), rabbit anti-GSK3 $\beta$ (Proteintech Group, Inc., 22104-1-AP, 1:1,000). Then, the membranes were incubated with HRP-conjugated goat anti-mouse IgG (BOSTER, BA1051, 1:50,000) or HRP-conjugated goat antirabbit IgG (BOSTER, BA1054, 1:50,000) for $2 \mathrm{~h}$ at $37^{\circ} \mathrm{C}$. The blots were visualized with Pierce ${ }^{\mathrm{TM}}$ ECL western blotting substrate (Thermo, NCI5079) and then exposed to X-ray films
(Kodak, XBT-1). The relative protein levels were quantified using Image $\mathrm{J}$ software (NIH, Bethesda, MD, USA).

\section{Statistical analysis}

All experiments were repeated at least 3 times. Data were recorded in Excel (Microsoft) and analyzed using GraphPad Prism 7 (GraphPad Software, La Jolla, USA). All data are presented as the mean $\pm \mathrm{SD}$. An unpaired $t$-test was used to analyze the difference between 2 groups and two-way ANOVA was used to identify differences between 3 or more groups.

\section{Results}

\section{Characterization of the OVX model}

Three months after establishment of the OVX model, MicroCT was used to quantify the microstructural parameters of the femurs in both groups. The results revealed that the distal femur in OVX was thinner than in the sham group (Figure 1A-D), which indicated the successful establishment of the osteoporotic rat model. Therefore, we isolated rBMSCs from both the sham and OVX groups. Flow cytometry analysis revealed that the cells were CD90+ CD29+ and CD34CD45- (Figure 2), which confirmed that the isolated cells were BMSCs.

\section{Characterization of rBMSCs after in vitro culture}

The BMSCs from both the sham and OVX groups were cultured through passage 10 . After primary seeding, passage 3 rBMSCs were employed for osteogenic induction. After being induced for 7 days, rBMSCs were stained with ALP, an early osteogenic differentiation marker, to check cell fates. The rBMSC derived from the sham group (sham-rBMSC hereinafter) exhibited intensive ALP staining 7 days after induction (Figure 3A, $\mathrm{A}^{\prime}$ ). However, the rBMSC derived from the OVX group (OVX-rBMSC hereinafter) secreted less ALP (Figure 3B, B'), which indicated that osteoblastic differentiation was significantly inhibited. The expression of periostin was significantly reduced in OVX-rBMSC compared to sham-rBMSC (Figure 3C). In contrast, primary rBMSCs were also induced for adipogenic differentiation. After a 14-day induction, the sham-rBMSC (Figure 3D, D') exhibited a much greater increased intensity of oil red $\mathrm{O}$ staining compared to OVX-rBMSC (Figure 3E, E'). Thus, adipogenic differentiation was significantly impaired after OVX treatment.

Table 1 - Primer sequences used for real-time PCR.

\begin{tabular}{lc}
\hline Gene & Sequence \\
\hline$\beta$-actin F & CACGATGGAGGGGCCGGACTCATC \\
$\beta$-actin R & TAAAGACCTCTATGCCAACACAGT \\
OCN F & CCTCTCTCTGCTCACTCTGCTG \\
OCN R & CTATTCACCACCTTACTGCCCTC \\
BSP F & CAGTTATGGCACCACGACAG \\
BSP R & CCATGCCCCTTGTAGTAGCT \\
Runx2 F & TCAGCGTCCTATCAGTTCCC \\
Runx2 R & ATTCAAAACGGTTGGGGAGC \\
periostin F & GTTCCTGTGTGACGTTGACC \\
periostin R & CGGGGCAGCATTCATATAGC \\
\hline
\end{tabular}

The relative quantification of gene expression was calculated using the $2^{-\Delta \Delta \mathrm{CT}}$ method normalized to $\beta$-actin gene expression. 

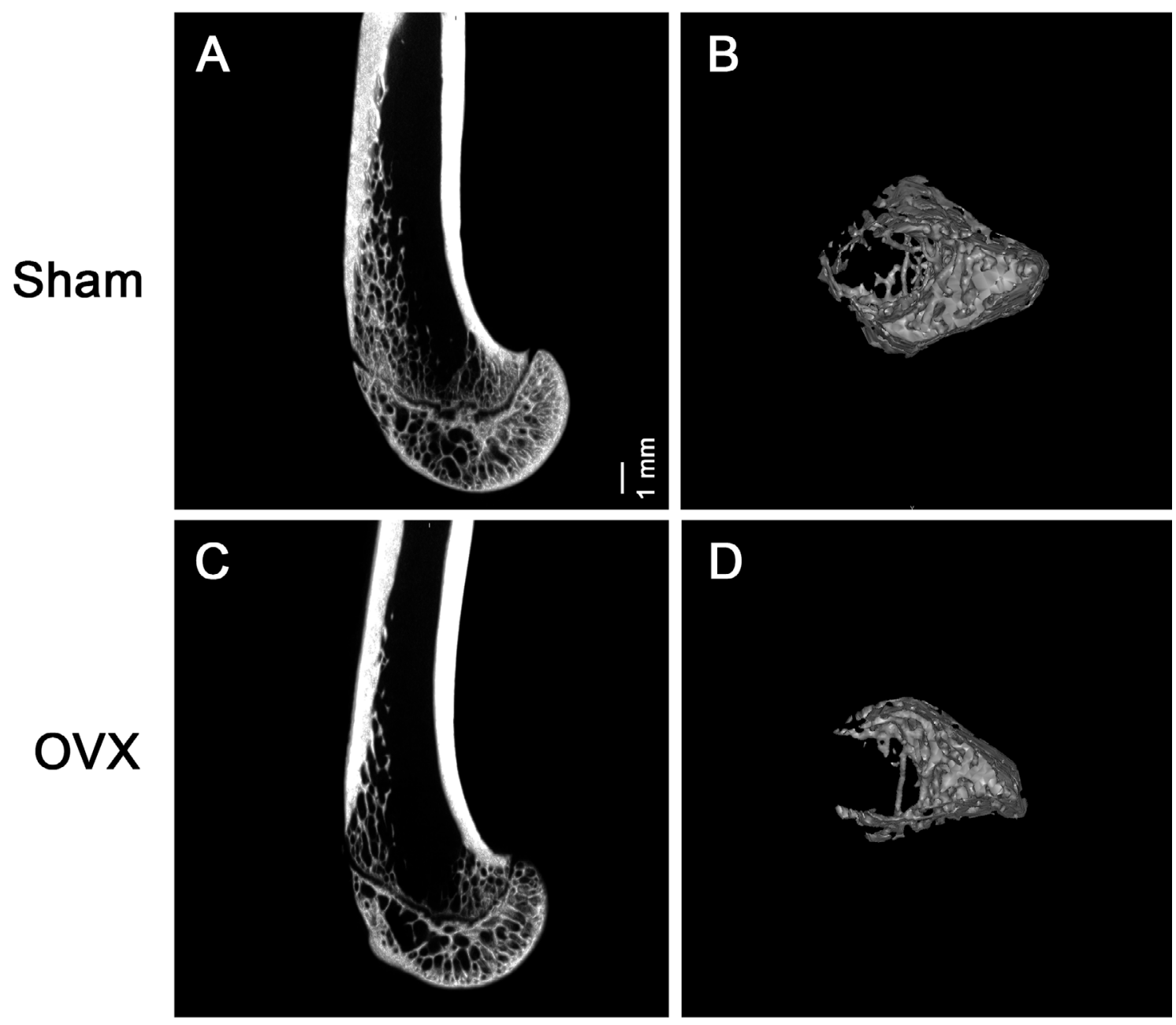

Figure 1 - Ovariectomy leads to osteoporosis in rats. (A-D) Representative MicroCT images of distal femurs. (A) and (B) are from rats in the sham group. (C) and (D) are from rats in the OVX group. Scale bar $=1 \mathrm{~mm}$.
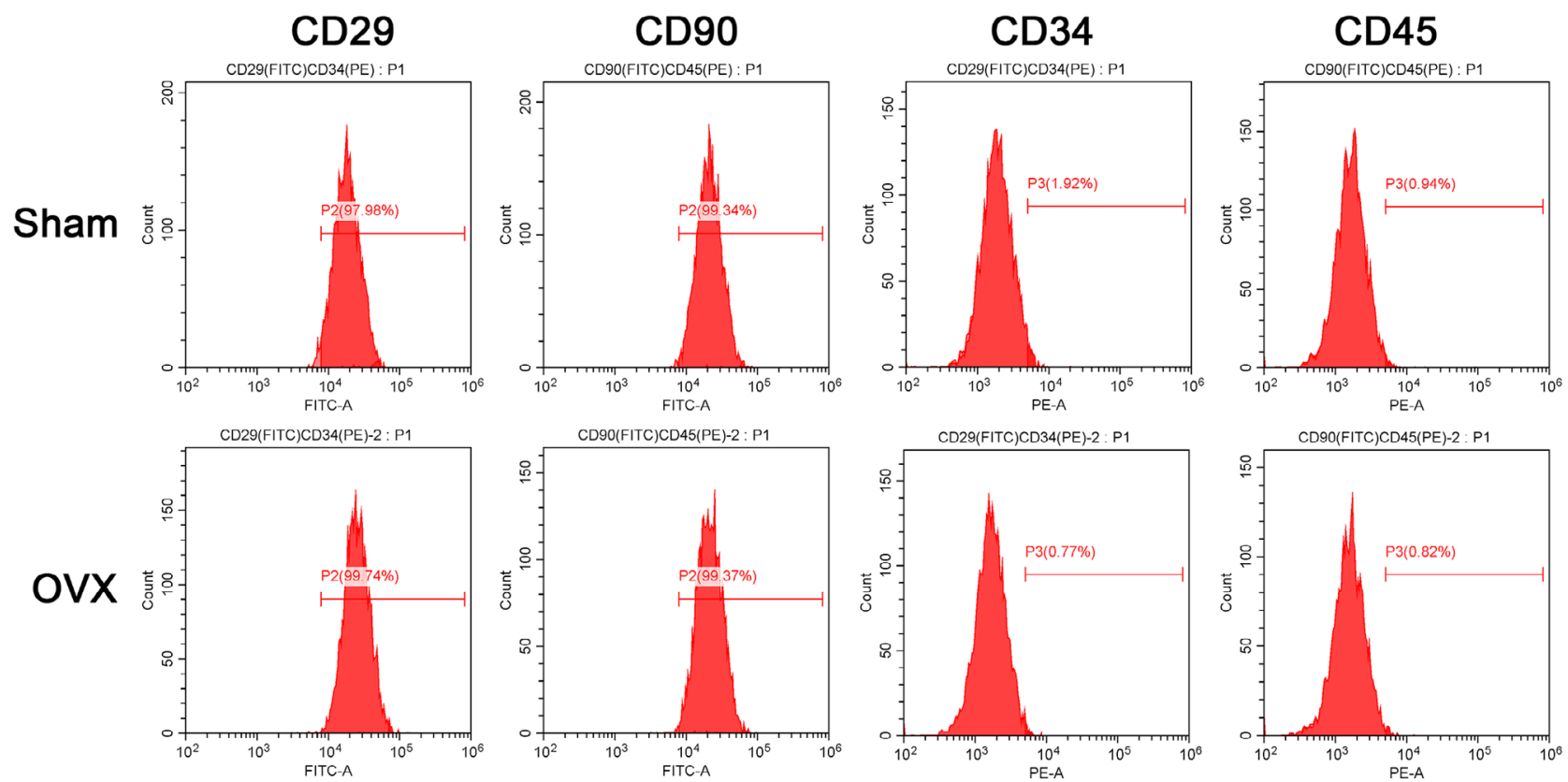

Figure 2 - Identification of BMSCs by flow cytometry analysis. Representative images of flow cytometry analysis. Sham-BMSCs were positive for the surface antigens CD29 (97.98\%) and CD90 (99.34\%) and negative for CD34 (1.92\%) and CD45 (0.94\%). OVX-BMSCs were positive for the surface antigens CD29 (97.74\%) and CD90 (99.37\%) and negative for CD34 (0.77\%) and CD45 (0.82\%). 


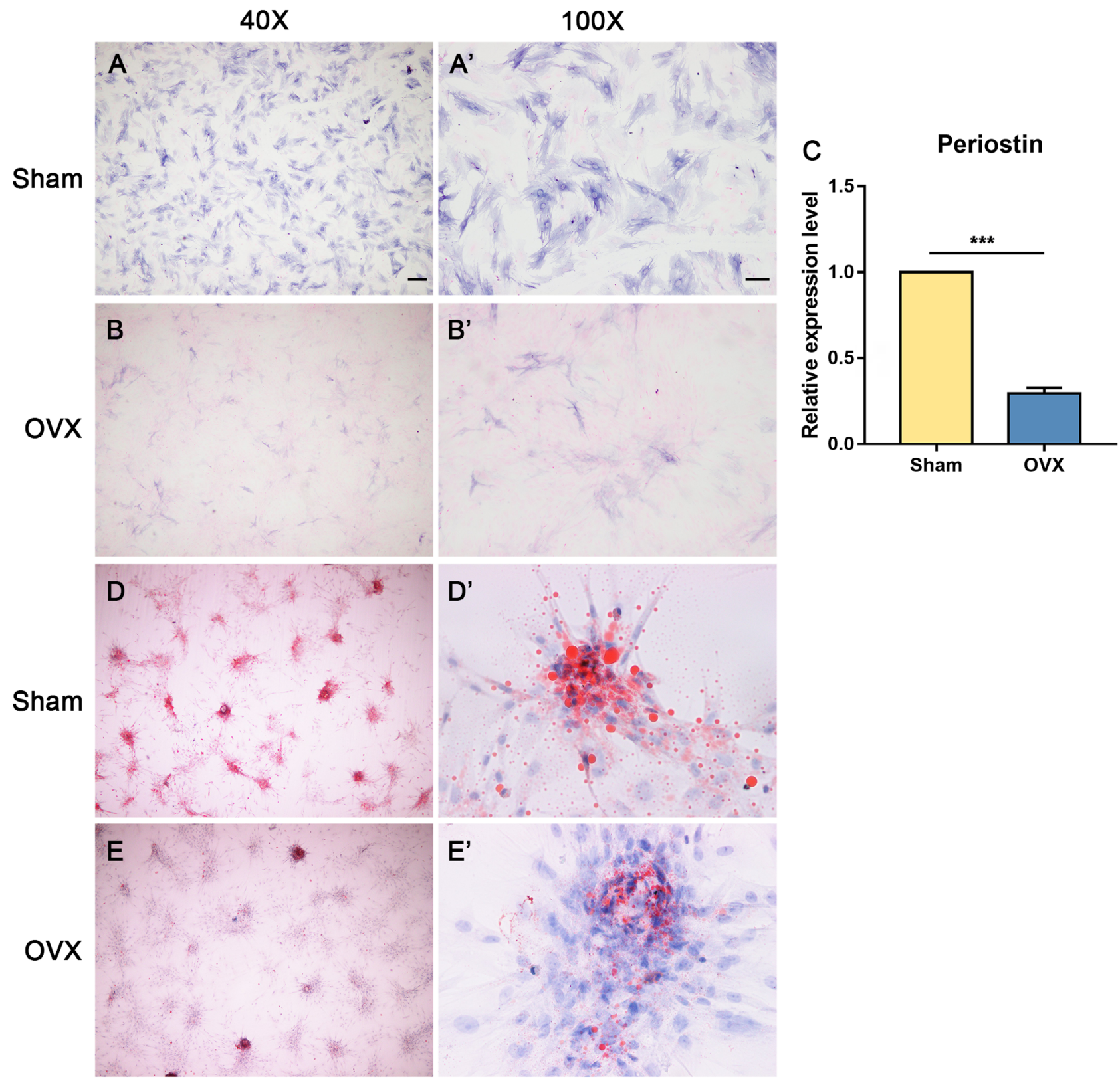

Figure 3 - BMSCs derived from OVX rats exhibited impaired osteogenesis ability, adipogenesis ability and reduced expression of periostin. (A-B') Representative images of ALP staining on day 7 after osteogenesis induction. (A, A') Sham-BMSCs express ALP, while (B, B') OVX-BMSCs express less ALP. (C) Graph shows the quantification of the relative expression level of periostin on day 7 after osteogenesis induction. Data are presented as the mean $\pm \mathrm{SD}$. ${ }^{* *} P<0.001$. (D-E') Representative images of oil red $\mathrm{O}$ staining on day 14 after adipogenesis induction. (D, D') Sham-BMSCs exhibited intracellular lipid droplets. (E, E') OVX-BMSCs exhibited less lipid droplets. The images are from 1 of 3 independent experiments. (A, B, D, E) Magnification $\times 40$, scale bar $=100 \mu \mathrm{m}$. (A', B', D', E') Magnification $\times 100$, scale bar $=200 \mu \mathrm{m}$.

\section{Periostin promotes rBMSCs osteoblastic differentiation}

Since we found a reduction in Periostin expression in rBMSCs derived from the osteoporosis model rats, we investigated its effects on rBMSC osteogenic differentiation. To overexpress periostin in rBMSC, lentivirus-mediated transfection was employed. $48 \mathrm{~h}$ after lentivirus transfection, the osteogenic induction medium was used for rBMSC culture. First, the transfection efficiency was evaluated on day 0 , day 7, day 14 and day 21 after transfection by qRT-PCR. Total RNA was isolated and the transcription level of periostin was determined. On day 0 , there was no significant difference in the periostin mRNA level among the 4 groups. However, on day 7 , day 14 and day 21 , periostin mRNA levels were reduced in
OVX-rBMSC and OVX-rBMSC transfected with empty vector (OVX-rBMSC-empty), compared to sham-rBMSC. rBMSCOVX transfected with periostin (OVX-rBMSC-periostin) significantly restored the periostin mRNA expression level (Figure 4A); the protein level of Periostin was determined using western blotting. In sham-rBMSC, the Periostin protein level was increased over time. In addition, Periostin protein was upregulated in OVX-rBMSC-periostin at each time point studied compared to OVX-rBMSC or OVX-rBMSC-empty. More importantly, as the osteogenic induction progressed, Periostin protein levels also increased in OVX-rBMSC-periostin (Figure 4B, C). These results indicated that the expression of Periostin could be induced after osteogenic induction in 

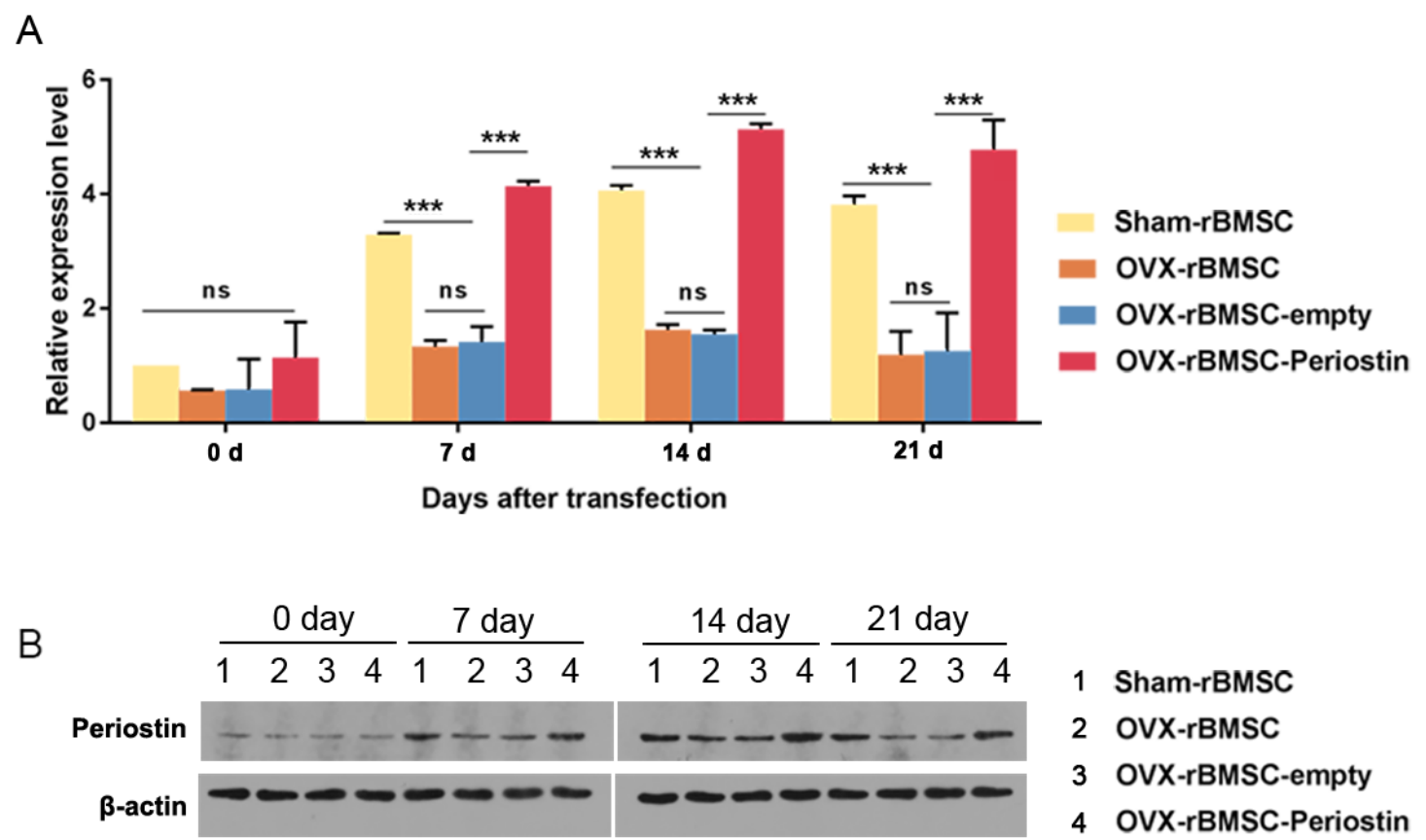

C

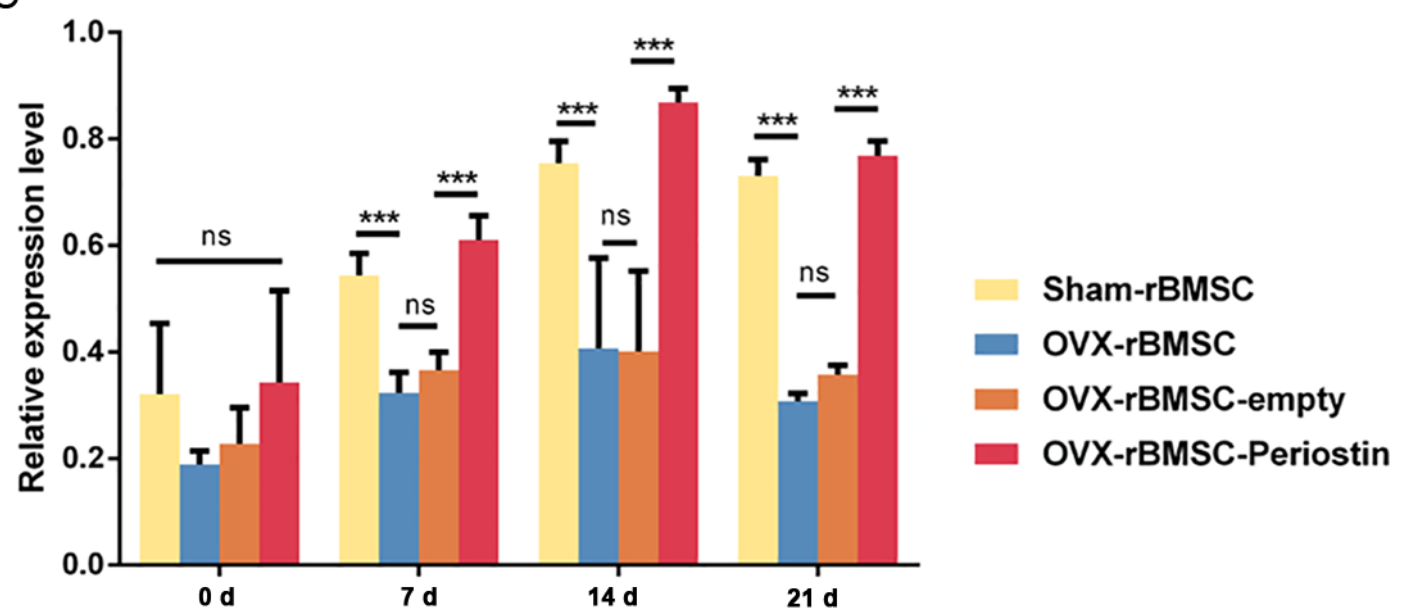

Figure 4-Overexpression of periostin elevated periostin mRNA and protein levels after osteogenesis induction. (A) The graph shows the quantification of the relative expression levels of periostin in the indicated groups on day 0 , day 7, day 14 and day 21 after osteogenesis induction. (B) The expression levels of periostin in the indicated groups on day 0 , day 7 , day 14 and day 21 after osteogenesis induction were investigated using western blotting; $\beta$-actin served as the reference gene. The image is representative of 3 independent experiments. (C) Quantitative western blotting was performed using Image $\mathrm{J}$ software. Data are presented as the mean $\pm \mathrm{SD}$ of 3 independent experiments. ns, not significant. ${ }^{* * *} P<0.001$; One-way ANOVA.

sham-rBMSC. Furthermore, lentivirus-mediated periostin overexpression could restore its expression, which was suppressed in OVX-rBMSC.

In order to assess the osteogenic differentiation ability of OVX-rBMSC-periostin, ALP staining was employed to evaluate the fate of cells. After being transfected with corresponding lentivirus vectors or untreated, cells of the different groups underwent osteogenic induction. ALP staining was assessed 7 days post induction. Overexpression of periostin significantly restored ALP activity and calcium mineralization formation in rBMSC-OVX (Figure 5A, B). Alizarin red S staining was used to monitor calcium deposit formation after a 21-day induction. Overexpression of periostin remarkably increased calcium nodule formation in rBMSC-OVX compared to the controls (Figure 6A, B). These results indicated that Periostin could promote osteoblastic differentiation in vitro.
Periostin regulates rBMSCs osteoblastic differentiation through the ILK/Akt/GSK3 $\beta$ pathway

It has been reported that ILK-deficient MC3T3-E1 cells exhibit a stronger mineralization ability (El-Hoss et $a l ., 2014$ ), indicating that ILK may slow down osteoblast maturation. On the other hand, Akt/GSK3 $\beta$ signaling also promotes osteoblast differentiation. Thus, we first evaluated ILK, Akt and GSK $3 \beta$ protein levels in the controls and OVXrBMSC-periostin using western blotting. Of note, ILK was significantly reduced in OVX-rBMSC in comparison to shamrBMSC. However, overexpression of periostin could restore ILK protein levels. Neither Akt nor GSK3 $\beta$ protein levels were altered in each group. Nonetheless, the phosphorylation of Akt and GSK3 $\beta$ were attenuated in OVX-rBMSC, as detected by antibodies against $\mathrm{pAKT}$ and $\mathrm{pGSK} 3 \beta$ and these phosphorylated proteins were relieved upon transfection 
with periostin in OVX-rBMSC (Figure 7A, rows 1-3). These results indicated that Periostin could regulate the activity of the ILK/Akt/GSK3 $\beta$ pathway. To elucidate whether Periostin regulates rBMSCs osteoblastic differentiation by action on the ILK/Akt/GSK3 $\beta$ pathway, rBMSC-OVX transfected with periostin were treated with the ILK inhibitor, OSU-T315 or the Akt inhibitor, genistein, before being stained with ALP to evaluate the fate of cells. First, we found that ILK and pAkt protein levels were significantly reduced after treatment with inhibitors, and subsequently that the level of pGSK $3 \beta$ was also reduced (Figure 7A, rows 3-5, B). Furthermore, the results of ALP and alizarin red S staining revealed that both inhibitors could reduce the osteoblastic differentiation of periostinOVX-rBMSC (Figure 8). Consistent with the above data, the qPCR results revealed reduced expressions of OCN, BSP and Runx2 after inhibitor treatment (Figure 7C). Interestingly, periostin was also downregulated at both the mRNA and protein level after ILK/Akt/GSK3 $\beta$ activity was blocked in periostin-OVX-rBMSC. These results indicated that Periostin was also regulated by ILK/Akt/GSK3 $\beta$ signaling activity.

A

Sham-rBMSC

OVX-rBMSC

OVX-rBMSC-empty

OVX-rBMSC-Periostin

$7 \mathrm{~d}$

$14 d$
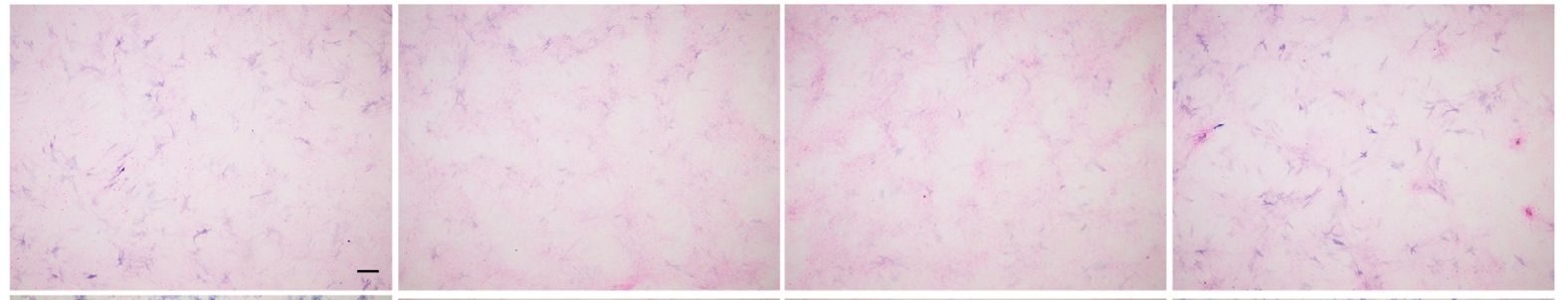

$21 d$
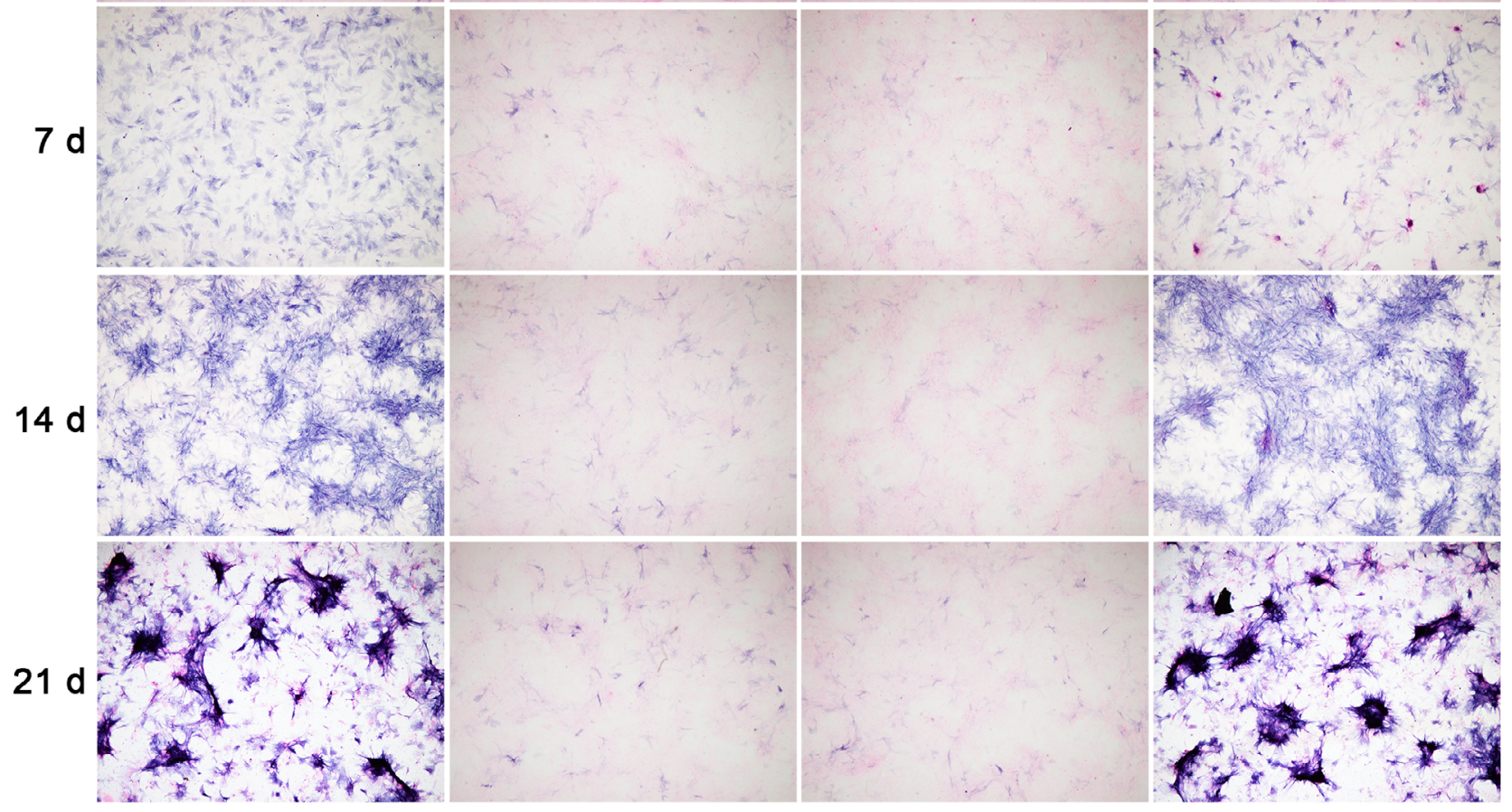

B
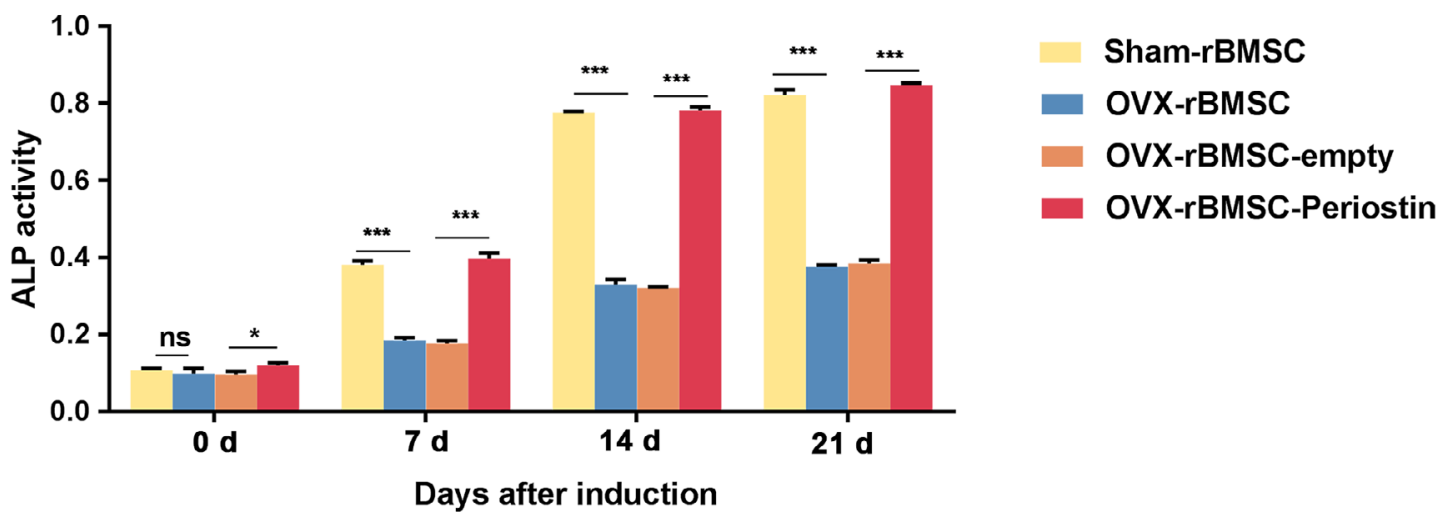

Figure 5 - Overexpression of periostin promotes ALP production by OVX-BMSCs. (A) Representative images of ALP staining of the indicated groups on day 0 , day 7 , day 14 and day 21 after osteogenesis induction. The images are from 1 of 3 independent experiments; Magnification $\times 40$, scale bar $=200 \mu \mathrm{m}$. (B) Graph shows quantitative analyses of ALP activity of the indicated groups on day 0 , day 7, day 14 and day 21 after osteogenesis induction. Data are presented as the mean $\pm \mathrm{SD}$ of 3 independent experiments. ns, not significant. $* P<0.05, * * * P<0.001$; One-way ANOVA. 


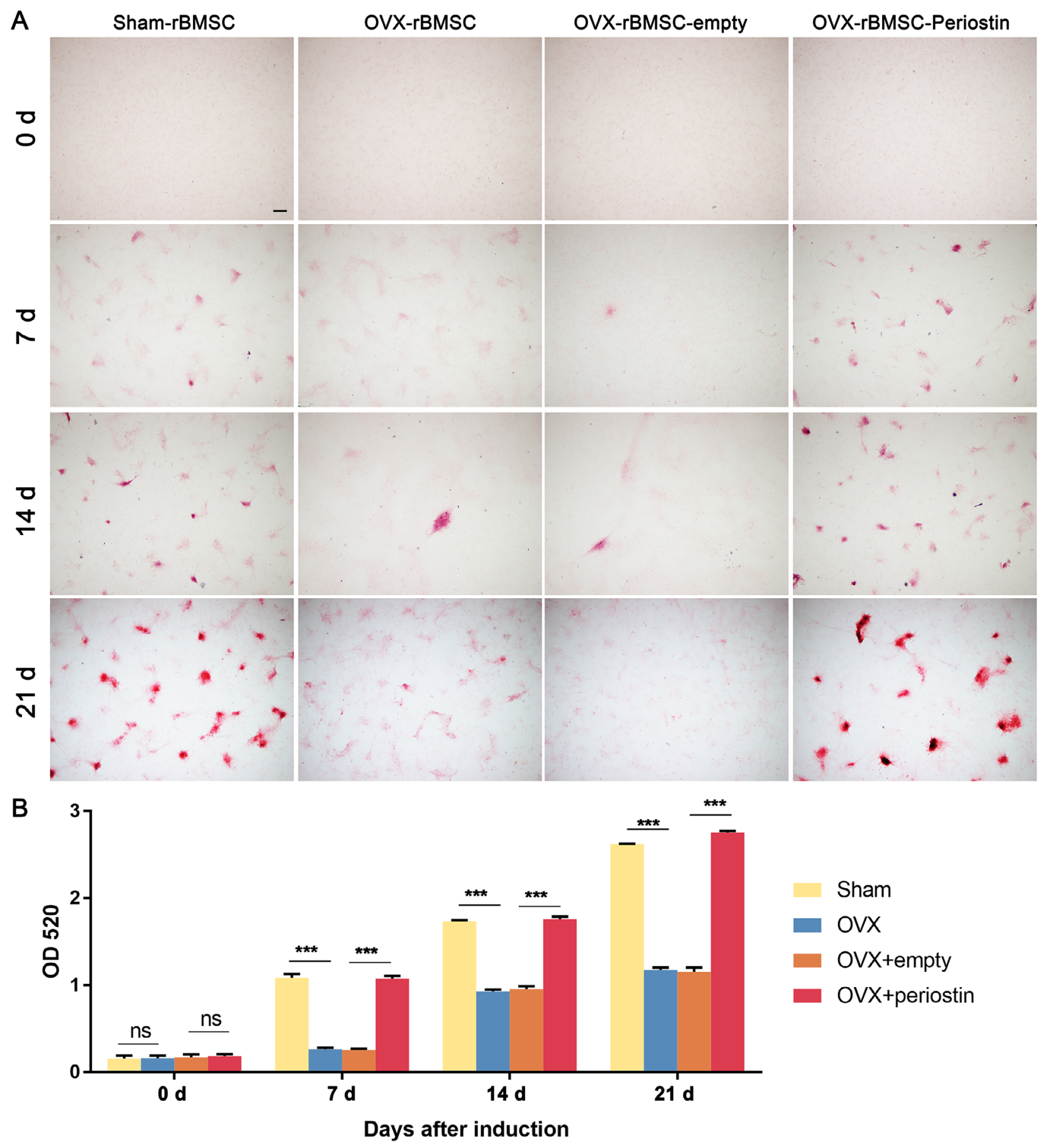

Figure 6 - Overexpression of periostin increased ARS staining in OVX-BMSCs. (A) Representative images of ARS staining of the indicated groups on day 0 , day 7 , day 14 and day 21 after osteogenesis induction. The images are from 1 of 3 independent experiments, magnification $\times 40$, scale bar $=200 \mu \mathrm{m}$. (B) Graph showing quantitative analyses of ARS of the indicated groups on day 0, day 7, day 14 and day 21 after osteogenesis induction. Data are presented as the mean $\pm \mathrm{SD}$ of 3 independent experiments. ns, not significant. $* * * P<0.001$; One-way ANOVA. 


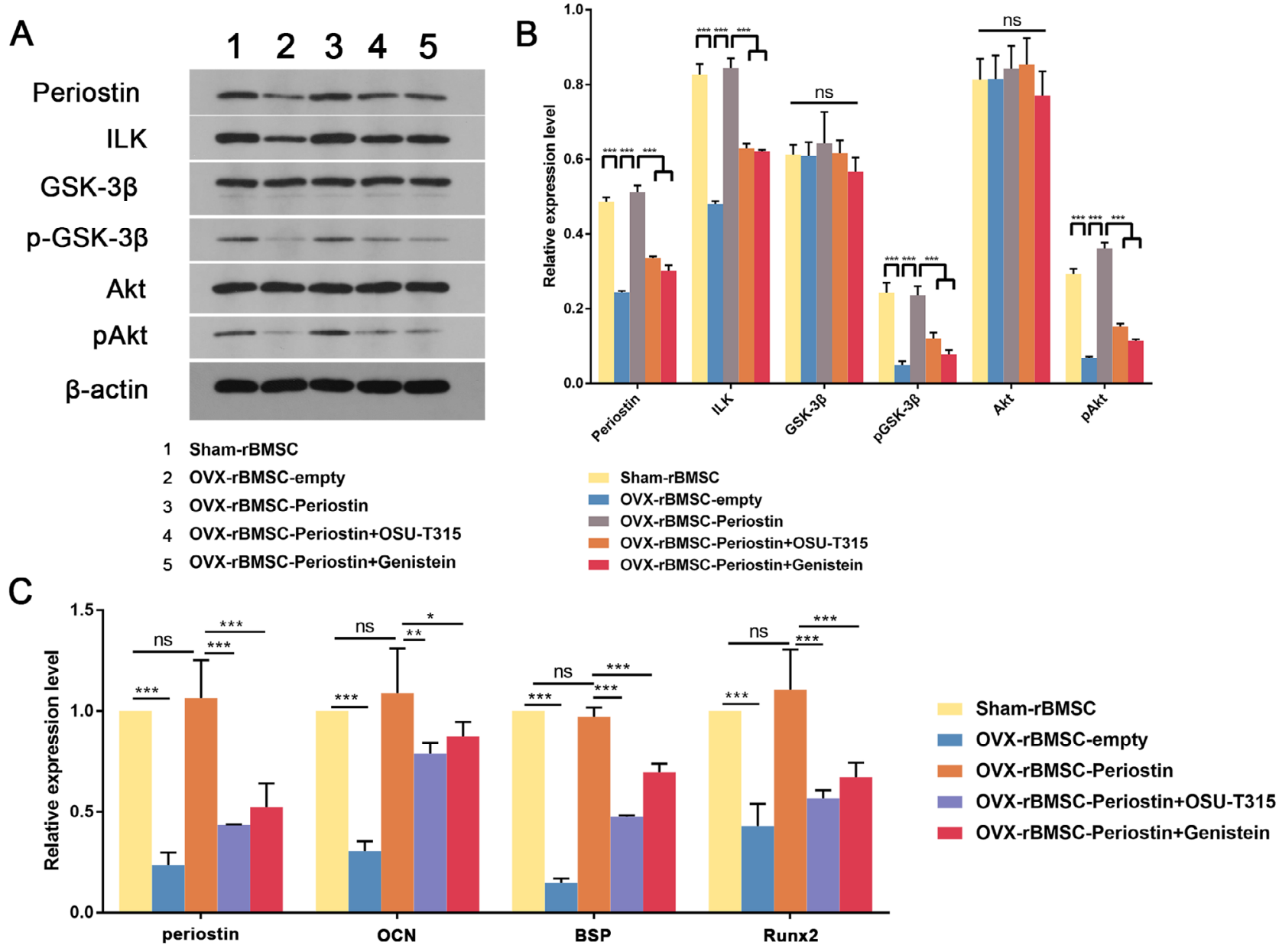

Figure 7 - Periostin regulates the expression of ILK, pAkt and GSK3 $\beta$ in OVX-BMSCs. (A) The expression levels of periostin, ILK, GSK3 $\beta$, pGSK3 $\beta$, Akt, pAkt in the indicated groups on day 7 after osteogenesis induction were examined by western blotting; $\beta$-actin served as the reference gene. (B) Quantitation of western blotting in (A) was performed using Image J software. (C) Graphs showing the relative mRNA expression levels of periostin, OCN, BSP and Runx 2 in the indicated groups on day 7 after osteogenesis induction. Data are presented as the mean \pm SD of 3 independent experiments. ns, not significant. $* P<0.05, * * P<0.01, * * * P<0.001$; One-way ANOVA.

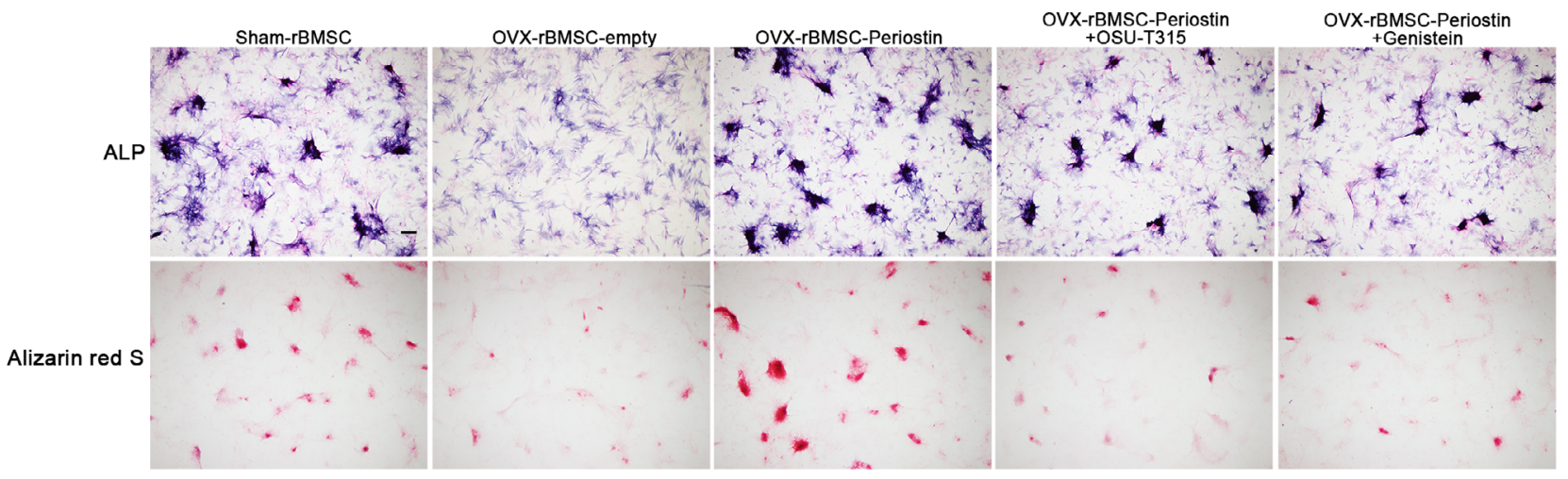

Figure 8 - Periostin promotes the osteogenesis ability of OVX-BMSCs in part by action on the ILK/Akt/GSK3 axis. (A) Representative images of ALP staining of the indicated groups on day 7 after osteogenesis induction. The images are from 1 of 3 independent experiments, magnification $\times 40$. (B) Representative images of ARS staining of the indicated groups on day 7 after osteogenesis induction, magnification $\times 40$, scale bar $=200 \mu$ m. 


\section{Discussion}

In bone, Periostin is a key ECM component of the periosteum. Moreover, it is known to promote chondrocyte differentiation by enhancing Akt phosphorylation via integrin as demonstrated in a 3D culture model (Inaki et al., 2018). In the present study, we found that Periostin was reduced in BMSCs derived from the osteoporosis rat model and that overexpression of periostin could enhance the osteogenic ability of BMSCs, in part by activating the ILK/Akt/GSK3 $\beta$ axis. In line with the expression level of Periostin, ILK, pAkt and $\mathrm{pGSK} 3 \beta$ levels were also reduced in BMSCs derived from female rats with osteoporosis. However, all of these three proteins recovered to the level equivalent to the control group after periostin overexpression. It is also worth noting that both mRNA and protein levels of Periostin were reduced when the ILK/Akt/GSK3 $\beta$ axis activity was suppressed, although periostin was simultaneously overexpressed by lentivirus. Thus, it is reasonable to conclude that the stability of periostin mRNA or Periostin protein was affected by the suppression of Akt/GSK3 $\beta$ signaling. Since Periostin expression was not completely abolished in BMSCs-OVX, we cannot rule out the possibility that the constitutive expression of Periostin was attenuated when the ILK/Akt/GSK3 $\beta$ axis was blocked. These results suggest a negative feedback regulation of Periostin activity in osteogenesis and that the underlying mechanisms involved require further investigation.

Osteoblast differentiation is tightly regulated by various soluble factors including systemic and local factors, which include the sex steroids and extracellular signals. In addition, mechanical stress is also essential for osteoblast differentiation. Long-term mechanical unloading, such as prolonged bed rest, immobilization or microgravity in space, can inhibit osteoblast differentiation (Jaworski et al., 1980), because mechanical stress is critical for osteoblast differentiation. Integrin is known for its ability to relay the mechanical cue from the environment to the nucleus through stress fiber formation (Burridge and Guilluy, 2016). More importantly, mechanical stress promotes osteogenic MC3T3-E1 cell activity via integrin- $\beta 1$-mediated $\beta$-catenin signaling (Yan et al., 2016). After binding to integrin, ILK has been reported to participate in the integrin-ERK $1 / 2$ cascade in the process of periodic mechanical stress-induced chondrocyte proliferation and matrix synthesis. It is noteworthy that the expression of ILK is also induced by mechanical stress (Song et al., 2016). In contrast, ILK has been reported to be involved in the phosphorylation of Akt and GSK3 $\beta$ (Liu et al., 2017). In our study, ILK, pAkt and pGSK $3 \beta$ were upregulated by Periostin. In addition, the levels of pAkt and pGSK $3 \beta$ were altered by modulation of Periostin, rather than AKT and GSK $3 \beta$. Thus, in the scenario of osteoblast differentiation, Periostin may regulate osteoblast differentiation through the mechanical transduction-based ILK/pAkt/pGSK3 $\beta$ axis.

The ILK/Akt/GSK3 $\beta$ axis has been reported to play roles in cancer cell growth and survival (Liu et al., 2017). Our results suggest a potential relationship between Periostin and ILK/Akt/ GSK3 $\beta$ in the process of BMSC differentiation. As an upstream regulator of GSK3 $\beta$, canonical Wnt/ $\beta$-catenin signaling plays a role in osteogenic differentiation (Kikuchi et al., 2007; Maupin et al., 2013). Similarity, in human ankylosing spondylitis, a chronic inflammatory disease, Periostin is downregulated and associated with the Wnt pathway (Solmaz et al., 2018). In CTLA4-modified MSCs, Periostin promoted osteogenic differentiation via the Wnt signaling pathway. Our study has provided molecular evidence that Periostin plays a key role in osteogenic differentiation via Wnt signaling in bone cells.

Since Periostin is a component of ECM proteins, it is able to communicate with the extracellular matrices. For example, in hematopoietic stem cells, Periostin can interact with Notch1 via CCN3 to maintain the hematopoietic stem cells (HSCs) or progenitor cells. More importantly, Notch pathway molecules, including Notch1, have been found to be increased in BMSCs derived from ovariectomized mice, which might participate in the prevention of osteogenic differentiation. Therefore, it is unlikely that Periostin promotes BMSC differentiation through the Notch pathway. Another extracellular matrix protein, bone morphogenetic protein-1 (BMP1), is also supported by Periostin activity. The interaction between BMP1/LOX1 and Periostin promotes collagen crosslinking, which may subsequently enhance TGF signaling activity. However, when human mesenchymal stem cells were incubated with BMP1/LOX1-modified cell matrices, the adipogenic differentiation ability of MSC was enhanced (Rosell-Garcia and Rodriguez-Pascual, 2018). Another study reported that upregulation of BMP1 promoted osteogenic differentiation (Zhang et al., 2019). Thus, whether Periostin can promote BMSC osteogenic ability through BMP1 and TGF pathways remains to be unequivocally established.

Previous studies have demonstrated that periostin can be regulated by estrogen. In human periodontal ligament cells, estrogen stimulation significantly increased periostin transcription. Our previous study also revealed that $17 \beta-E 2$ upregulated periostin expression in ovariectomized rats. Estrogen plays a protective role in bone formation and homeostasis; thus, it is also used as a drug to reduce bone loss. To date, most research on bone formation has concentrated on the characterization of BMSCs. whereas there is a paucity of studies that used BMSCs derived from postmenopausal osteoporosis model rats. First, the rodent postmenopausal osteoporosis model established by ovariectomy only reflects acute effects of estrogen deprivation. Although the osteogenic ability of BMSCs derived from OVX-rats is impaired in several passages in vitro, it is no longer regulated by systemic estrogen. Thus, we cannot conclude that the promotion of osteogenic ability by Periostin is under the control of estrogen. Second, it has been hypothesized that stem cells from the periosteum and bone marrow play distinct roles and bone modeling and remodeling benefit more from stem cells in the periosteum than those in the bone marrow (Duchamp de Lageneste et al., 2018). Third, specialized niche, which could not be mimicked in cell line-based studies, are essential for proliferation, survival and differentiation of skeleton stem cells. Altogether, more in vivo and in vitro studies are necessary to provide new insights into the molecular pathology of Periostin-related postmenopausal osteoporosis.

In summary, our findings have shown that Periostin can promote BMSC osteogenic differentiation through the ILK/ pAkt/pGSK $3 \beta$ axis in BMSCs derived from OVX-rats. Our findings suggest that Periostin may serve as a therapeutic 
target of osteoporosis. In subsequent research, in vivo Periostin knock-out experiments will be needed to confirm further its importance in the osteogenic effect in postmenopausal osteoporosis rat models.

\section{Acknowledgements}

This research was supported by National Natural Science Foundation of China (grant number 81470774) and Research Foundation for Youths of Chifeng College (grant number cfxyqn202147). The funders had no role in the design of the study or the collection, analysis and interpretation of data, or in writing the manuscript.

\section{Conflict of Interest}

The authors declare that there is no conflict of interest that could be perceived as prejudicial to the impartiality of the reported research.

\section{Author Contributions}

All of the authors have read and approved the manuscript. The authors were solely responsible for the conception and implementation of the study and for writing the manuscript. Conceptualization: ZLJ, MC; data collection: CRL, WWZ, DDL; analysis and interpretation: SLL; statistical analysis: SLL; writing original draft: SLL; writing review \& editing: DDH.

\section{References}

Bennett CN, Ouyang H, Ma YL, Zeng Q, Gerin I, Sousa KM, Lane TF, Krishnan V, Hankenson KD and MacDougald OA (2007) Wnt $10 \mathrm{~b}$ increases postnatal bone formation by enhancing osteoblast differentiation. J Bone Miner Res 22:1924-1932.

Bonnet N, Conway SJ and Ferrari SL (2012) Regulation of beta catenin signaling and parathyroid hormone anabolic effects in bone by the matricellular protein periostin. Proc Natl Acad Sci U S A 109:15048-15053.

Bonnet N, Gineyts E, Ammann P, Conway SJ, Garnero P and Ferrari S (2013) Periostin deficiency increases bone damage and impairs injury response to fatigue loading in adult mice. PLoS One 8:e78347.

Burridge K and Guilluy C (2016) Focal adhesions, stress fibers and mechanical tension. Exp Cell Res 343:14-20.

Byun MR, Hwang J-H, Kim AR, Kim KM, Hwang ES, Yaffe MB and Hong J-H (2014) Canonical Wnt signalling activates TAZ through PP1A during osteogenic differentiation. Cell Death Differ 21:854-863.

Chen G, Deng C and Li Y-P (2012) TGF-beta and BMP signaling in osteoblast differentiation and bone formation. Int J Biol Sci 8:272-288.

Deng Z-L, Sharff KA, Tang N, Song W-X, Luo J, Luo X, Chen J, Bennett E, Reid R, Manning D, et al. (2008) Regulation of osteogenic differentiation during skeletal development. Front Biosci 13:2001-2021.

Duchamp de Lageneste O, Julien A, Abou-Khalil R, Frangi G, Carvalho C, Cagnard N, Cordier C, Conway SJ and Colnot C (2018) Periosteum contains skeletal stem cells with high bone regenerative potential controlled by Periostin. Nat Commun 9:773.

Eastell R, O'Neill TW, Hofbauer LC, Langdahl B, Reid IR, Gold DT and Cummings SR (2016) Postmenopausal osteoporosis. Nat Rev Dis Primers 2:16069.

El-Hoss J, Arabian A, Dedhar S and St-Arnaud R (2014) Inactivation of the integrin-linked kinase (ILK) in osteoblasts increases mineralization. Gene 533:246-252.
Friedlander AH (2002) The physiology, medical management and oral implications of menopause. J Am Dent Assoc 133:73-81.

Gao Y, Huang E, Zhang H, Wang J, Wu N, Chen X, Wang N, Wen S, Nan G, Deng F, et al. (2013) Crosstalk between Wnt/ beta-catenin and estrogen receptor signaling synergistically promotes osteogenic differentiation of mesenchymal progenitor cells. PLoS One 8:e82436.

Gimble JM, Zvonic S, Floyd ZE, Kassem M and Nuttall ME (2006) Playing with bone and fat. J Cell Biochem 98:251-266.

Heo SC, Shin WC, Lee MJ, Kim BR, Jang IH, Choi EJ, Lee JS and Kim JH (2015) Periostin accelerates bone healing mediated by human mesenchymal stem cell-embedded hydroxyapatite/ tricalcium phosphate scaffold. PLoS One 10:e0116698.

Inaki R, Fujihara Y, Kudo A, Misawa M, Hikita A, Takato T and Hoshi K (2018) Periostin contributes to the maturation and shape retention of tissue-engineered cartilage. Sci Rep 8:11210.

Jaworski ZF, Liskova-Kiar M and Uhthoff HK (1980) Effect of long-term immobilisation on the pattern of bone loss in older dogs. J Bone Joint Surg Br 62-B:104-110.

Kikuchi A, Yamamoto H and Kishida S (2007) Multiplicity of the interactions of Wnt proteins and their receptors. Cell Signal 19:659-671.

Koh SJ, Kim JW, Kim BG, Lee KL, Kim DW and Kim JS (2019) Matricellular protein periostin promotes colitis-associated colon tumorigenesis in mice. Carcinogenesis 40:102-111.

Li D, Zhou W and Cao M (2019) Periostin-modified bone marrow mesenchymal stem cells from osteoporotic rats promote alveolar bone regeneration. J Mol Histol 50:493-502.

Liu L, Zhang S, Hu L, Liu L, Guo W and Zhang J (2017) HMGA1 participates in MHCC97H cell proliferation and invasion through the ILK/Akt/GSK3beta signaling pathway. Mol Med Rep 16:9287-9294.

Ma H, Wang J, Zhao X, Wu T, Huang Z, Chen D, Liu Y and Ouyang G (2020) Periostin promotes colorectal tumorigenesis through integrin-FAK-Src pathway-mediated YAP/TAZ activation. Cell Rep 30:793-806.e6.

Ma Z, Zhao X, Deng M, Huang Z, Wang J, Wu Y, Cui D, Liu Y, Liu R and Ouyang G (2019) Bone marrow mesenchymal stromal cell-derived periostin promotes B-ALL progression by modulating CCL2 in leukemia cells. Cell Rep 26:1533-1543.e4.

Maupin KA, Droscha CJ and Williams BO (2013) A comprehensive overview of skeletal phenotypes associated with alterations in Wnt/beta-catenin signaling in humans and mice. Bone Res 1:27-71.

Meunier P, Aaron J, Edouard C and Vignon G (1971) Osteoporosis and the replacement of cell populations of the marrow by adipose tissue a quantitative study of 84 iliac bone biopsies. Clin Orthop Relat Res 80:147-154.

Norris RA, Damon B, Mironov V, Kasyanov V, Ramamurthi A, Moreno-Rodriguez R, Trusk T, Potts JD, Goodwin RL, Davis $\mathrm{J}$, et al. (2007) Periostin regulates collagen fibrillogenesis and the biomechanical properties of connective tissues. J Cell Biochem 101:695-711.

O'Dwyer DN and Moore BB (2017) The role of periostin in lung fibrosis and airway remodeling. Cell Mol Life Sci 74:4305-4314.

Ohno F, Nakahara T, Kido-Nakahara M, Ito T, Nunomura S, Izuhara K and Furue M (2019) Periostin links skin inflammation to melanoma progression in humans and mice. Int $\mathrm{J}$ Mol Sci 20:169.

Rosell-Garcia T and Rodriguez-Pascual F (2018) Enhancement of collagen deposition and cross-linking by coupling lysyl oxidase with bone morphogenetic protein-1 and its application in tissue engineering. Sci Rep 8:10780.

Ross DA, Rao PK and Kadesch T (2004) Dual roles for the notch target gene Hes-1 in the differentiation of 3T3-L1 preadipocytes. Mol Cell Biol 24:3505-3513. 
Ryu G and Kim DW (2020) Th2 inflammatory responses in the development of nasal polyps and chronic rhinosinusitis. Curr Opin Allergy Clin Immunol 20:1-8.

Solmaz D, Uslu S, Kozaci D, Karaca N, Bulbul H, Tarhan EF, Ozmen M, Can G and Akar S (2018) Evaluation of periostin and factors associated with new bone formation in ankylosing spondylitis: Periostin may be associated with the Wnt pathway. Int J Rheum Dis 21:502-509.

Song B-Q, Chi Y, Li X, Du W-J, Han Z-B, Tian J-J, Li J-J, Chen F, Wu H-H, Han L-X, et al. (2015) Inhibition of Notch signaling promotes the adipogenic differentiation of mesenchymal stem cells through autophagy activation and PTEN-PI3K/AKT/ mTOR pathway. Cell Physiol Biochem 36:1991-2002.

Song H, Liang W, Xu S, Li Z, Chen Z, Cui W, Zhou J, Wang Q, Liu $\mathrm{F}$ and Fan W (2016) A novel role for integrin-linked kinase in periodic mechanical stress-mediated ERK1/2 mitogenic signaling in rat chondrocytes. Cell Biol Int 40:832-839.

Spinella-Jaegle S, Rawadi G, Kawai S, Gallea S, Faucheu C, Mollat P, Courtois B, Bergaud B, Ramez V, Blanchet AM, et al. (2001) Sonic hedgehog increases the commitment of pluripotent mesenchymal cells into the osteoblastic lineage and abolishes adipocytic differentiation. J Cell Sci 114:2085-2094.
Stevens JR, Miranda-Carboni GA, Singer MA, Brugger SM, Lyons KM and Lane TF (2010) Wnt10b deficiency results in agedependent loss of bone mass and progressive reduction of mesenchymal progenitor cells. J Bone Miner Res 25:2138-2147.

Yan Y, Sun H, Gong Y, Yan Z, Zhang X, Guo Y and Wang Y (2016) Mechanical strain promotes osteoblastic differentiation through integrin-beta1-mediated beta-catenin signaling. Int J Mol Med 38:594-600.

Zhang F, Luo K, Rong Z, Wang Z, Luo F, Zhang Z, Sun D, Dong S, Xu J and Dai F (2017) Periostin upregulates Wnt/betacatenin signaling to promote the osteogenesis of CTLA4modified human bone marrow-mesenchymal stem cells. Sci Rep 7:41634.

Zhang Y, Chen B, Li D, Zhou X and Chen Z (2019) LncRNA NEAT1/ miR-29b-3p/BMP1 axis promotes osteogenic differentiation in human bone marrow-derived mesenchymal stem cells. Pathol Res Pract 215:525-531.

Associate Editor: Catarina Satie Takahashi

License information: This is an open-access article distributed under the terms of the Creative Commons Attribution License (type CC-BY), which permits unrestricted use, distribution and reproduction in any medium, provided the original article is properly cited. 